\title{
4 × 4 MIMO Multiband Antenna System for Mobile Handsets
}

\author{
Le Huy Trinh, ${ }^{1}$ Fabien Ferrero, ${ }^{2}$ Leonardo Lizzi, ${ }^{2}$ Jean-Marc Ribero, ${ }^{2}$ and Robert Staraj ${ }^{2}$ \\ ${ }^{1}$ University of Information Technology, National University Ho Chi Minh City, Ho Chi Minh City, Vietnam \\ ${ }^{2}$ University of Nice Sophia Antipolis, CNRS, LEAT, Campus SophiaTech, Bâtiment Forum, 930 route des Colles, \\ BP 145, 06903 Sophia Antipolis, France
}

Correspondence should be addressed to Leonardo Lizzi; leonardo.lizzi@unice.fr

Received 2 September 2015; Revised 25 October 2015; Accepted 1 November 2015

Academic Editor: Miguel Ferrando Bataller

Copyright (c) 2015 Le Huy Trinh et al. This is an open access article distributed under the Creative Commons Attribution License, which permits unrestricted use, distribution, and reproduction in any medium, provided the original work is properly cited.

A $4 \times 4$ MIMO multiband antenna system for mobile handsets is proposed in this paper. The placement of the elements on the terminal chassis as well as the single structure has been optimized in order to reduce the Envelope Correlation Coefficient (ECC). Simulated and measured results, including also the integration with a real 7-inch commercial tablet, aimed at validating the proposed solution are presented.

\section{Introduction}

Emerging applications for mobile Internet access such as video or audio streaming require constantly improving mobile network data rates. In order to follow this trend, new technologies as carrier aggregation and Multiple Input Multiple Output (MIMO) need to be used. Intra- and Interband carrier aggregation imply that multiband behavior needs to be supported permanently, which limits the use of reconfigurable solutions. MIMO technology requires integrating several radiating elements in a terminal with low mutual coupling. For example, Long Term Evolution (LTE) release 10 enables up to $4 * 4$ MIMO schemes for uplink transmission; then, several elements need to be used.

Thus, one of the key challenges in mobile multiantenna systems is to design miniature and multiband elements, also having low Envelope Correlation Coefficients (ECC) and high efficiencies.

Recently, several solutions have been proposed to address this issue. In [1], a $125 \times 50 \times 1 \mathrm{~mm}$ reconfigurable structure limited to two elements integrated in a $260 \times 200 \mathrm{~mm}$ chassis has been proposed. Promising results are obtained in the $700-960 \mathrm{MHz}$ frequency band, but coverage in the higher band is limited to the LTE2500 standard $(2.5-2.7 \mathrm{GHz})$. In [2], an interesting study on a smart-phone with two different structures is proposed, but this work only focuses on bands higher than $1 \mathrm{GHz}$. In terms of antenna isolation in MIMO structures, several techniques have been also proposed to reduce ECC. A first solution was proposed in [3] for a 4element system based on the neutralization line technique. The isolation improvement is very important $(>10 \mathrm{~dB})$ but operates only on a single and narrow band. Several works have proposed adding slots in the PCB to realize wave-traps $[4,5]$. All these different techniques improve antenna isolation but increase the complexity of the terminal fabrication, especially when the tablet edge has to be used to place several connectors, sensors, and speakers.

In order to take into account all these constraints, a proper methodology has to be used. One of the most used approaches relies on the study of the characteristic modes of the structure $[6,7]$. The main objective of this approach is to place the antenna elements on the structure in such a way that each element excites the fundamental modes with different amplitudes and phases. As a result, the multiantenna system will have a low ECC.

In this paper, we propose a 4-radiating-element MIMO system solution enabling multiband behavior, low ECC, and high efficiency on a small terminal having limited volume dedicated to the antenna elements. The design methodology consists in two phases: the first one is the choice of the position of the elements on the terminal chassis, and the second one is the design of a specific multiband structure suitable for the selected position. 


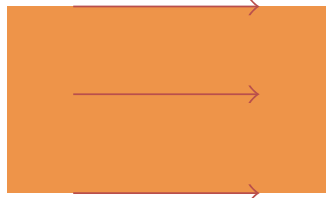

$J 1$

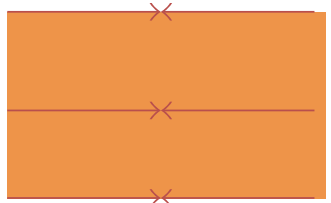

J3

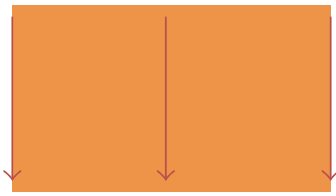

J2

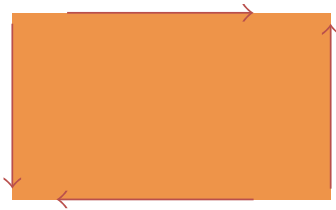

J4
FIGURE 1: Characteristic modes of a ractangular plate.

\section{Multiantenna System Design}

2.1. Element Placement. Concerning ECC in mobile communication technology, the main issues appear in the lower frequency $(<1 \mathrm{GHz})$ because of the large wavelength compared to the terminal size. It has been demonstrated that, in this frequency range, the main part of the radiation comes from the chassis [8]. When a rectangular plate is considered, the first 4 characteristic modes excited are presented in Figure 1 [9]. For capacitive excitation performed by classical electric resonators like monopoles, the position to excite a specific mode has to be chosen where the current is minimal. Considering this fact, the only position on the chassis that can be used to excite the four modes simultaneously is one of the 4 plate corners. This configuration can be used for $4 \times 4$ MIMO systems because it allows the integration of 4 identical elements and maximizes the spacing among them. Consequently, a specific element structure has been designed to fit this configuration.

2.2. Single Element Design. Dealing with the design of antennas for portable devices, three main challenges have to be faced: miniaturization, impedance matching, and acceptable efficiency over all the requested frequency bands. In latest mobile terminals, required standards are usually LTE 700 (790-862 MHz), GSM (880-960 MHz), DCS (1710-1880 MHz), PCS (1850-1990 MHz), UMTS 2100 (1920-2170 MHz), WLAN (2400-2480), LTE 2600 (2500$2690 \mathrm{MHz}$ ), and LTE $3500(3400-3600 \mathrm{MHz})$. In order to simultaneously cover all these different frequencies, complex antenna structures are needed.

The proposed structure is based on a folded monopole structure coupled with parasitic elements. The folded monopole has been widely used thanks to the small dimension and low-cost production and since it allows both the decrease of its size and the control of the higher resonance modes. Figures 2(a) and 2(b) show the geometry of the proposed antenna. It includes a two-arm folded radiating element and two parasitic elements. The proposed antenna is mounted on a $130 \times 190 \mathrm{~mm}^{2}$ ground plane, typical of midsize mobile devices as shown in Figure 2(c).
TABLE 1: Optimized values of the antenna geometrical parameters (in $\mathrm{mm}$ ).

\begin{tabular}{ccccccccccccccc}
\hline$L 1$ & L2 & L3 & L4 & L5 & L6 & L7 & L8 & H1 & $H 2$ & $H 3$ & $W 1$ & $W 2$ & $W 3$ & $W 4$ \\
\hline 31 & 8 & 32 & 20 & 13 & 12 & 14 & 3.5 & 8 & 4.5 & 7 & 4 & 2 & 1.5 & 4 \\
\hline
\end{tabular}

To understand the proposed antenna radiation mechanism, a parametric study is presented to explain the influence of the different parameters. The longer folded monopole has a length of about $100 \mathrm{~mm}$ (mainly $L 1+L 2+L 3$ ), equal to $\lambda_{1} / 4, \lambda_{1}$ being the wavelength at $750 \mathrm{MHz}$, allowing the low frequency band to be covered. By changing $L 1$, the center frequency of this low band can be tuned as shown in Figure 3.

The folded shape of this branch also allows the decrease of the third-order resonance from $2250 \mathrm{MHz}$ to $1700 \mathrm{MHz}$. The shift amount is controlled by the coupling between $L 1$ and $L 3$, as shown in Figure 4, where the effects given by the $L 3$ variation are reported. To extend the middle band up to 2.7 GHz, a parasitic ground strip is placed close to the feeding port. Its total length $H 1+L 6+H 3$ equals $28 \mathrm{~mm}$, which corresponds to a quarter wavelength at $2.7 \mathrm{GHz}$. The coupled resonance can be tuned by varying $\mathrm{H} 3$ as shown in Figure 5. However, a frequency band from $1.7 \mathrm{GHz}$ to $2.7 \mathrm{GHz}$ is quite large and the combination of the two previous resonators is not enough to cover the entire band. To solve this issue, an additional arm in the folded inverted- $L$ shape of length $L 4+L 5$ is used. By tuning the length $L 5,-6 \mathrm{~dB}$ matching on the $1.7-2.7 \mathrm{GHz}$ is achieved as presented in Figure 6. An additional printed monopole of length $L 7+L 8$ is integrated in the design to cover the 3.4-3.6 GHz band. By changing the L7 length, the resonance can be tuned as shown in Figure 7.

The optimized geometrical parameters of the final antenna structure are presented in Table 1. Thanks to the symmetry of the structure, the simulated results are only presented in terms of $S 1 j$-parameters, $j=1, \ldots, 4$ in Figure 8 . A $-6 \mathrm{~dB}$ matching is obtained for the operating frequencies in the low $(740-960 \mathrm{MHz})$, middle $(1710-2690 \mathrm{MHz})$, and high bands $(3400-3600 \mathrm{MHz})$. Moreover, the maximum mutual coupling $(-7.8 \mathrm{~dB})$ is obtained at $800 \mathrm{MHz}$ between Antennas 1 and 4 (S14) because of their proximity. For the other combinations (S12 and S13) and bands, the antenna system exhibits an isolation better than $10 \mathrm{~dB}$.

\section{Numerical and Experimental Validation}

3.1. Analysis of the Current on the Structure and Simulated ECC. Figure 9 shows the current on the PCB at $800 \mathrm{MHz}$ when antenna 1 (up-left of the corner) is fed, and the other antennas are matched to $50 \mathrm{ohm}$. The antenna excites the different characteristic modes of the chassis. In the middle of the chassis, it can be seen that $J 1$ and $J 2$ modes are simultaneously excited, resulting in diagonal currents. The influence of the $J 3$ mode is visible in the center of the PCB where a null in the current density is observed along a line, which is slightly slanted with respect to Figure 1. From the sense of the current on the edges of the chassis, it can be deduced that the J4 mode is strongly excited. 


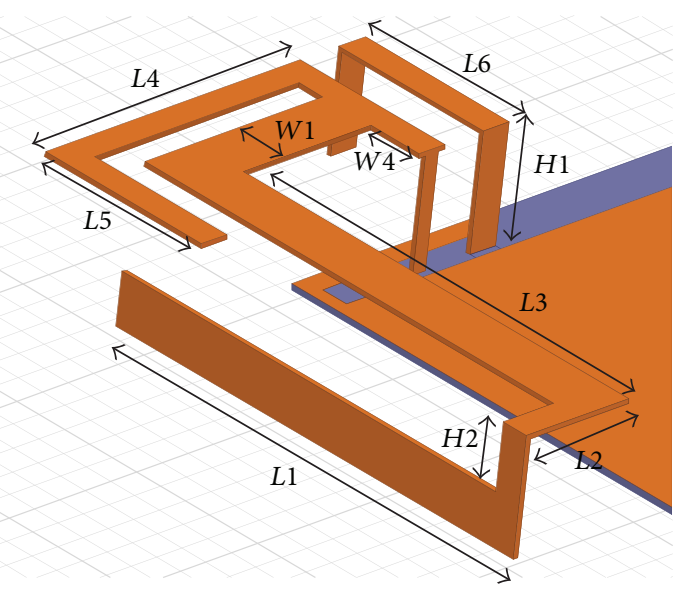

(a)

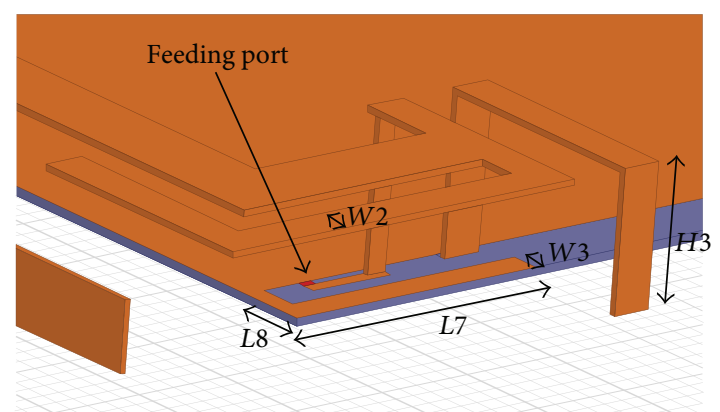

(b)

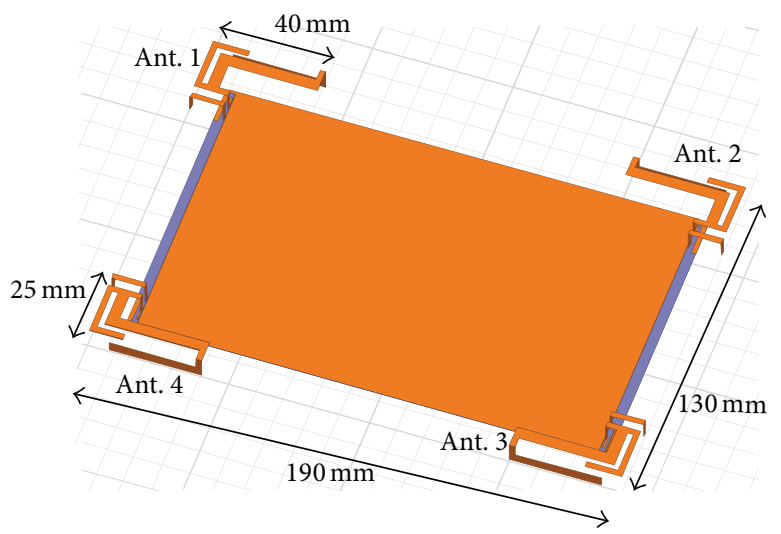

(c)

FIGURE 2: ((a) and (b)) 3D views of the single element and (c) view of the terminal integrating the 4 antenna elements.

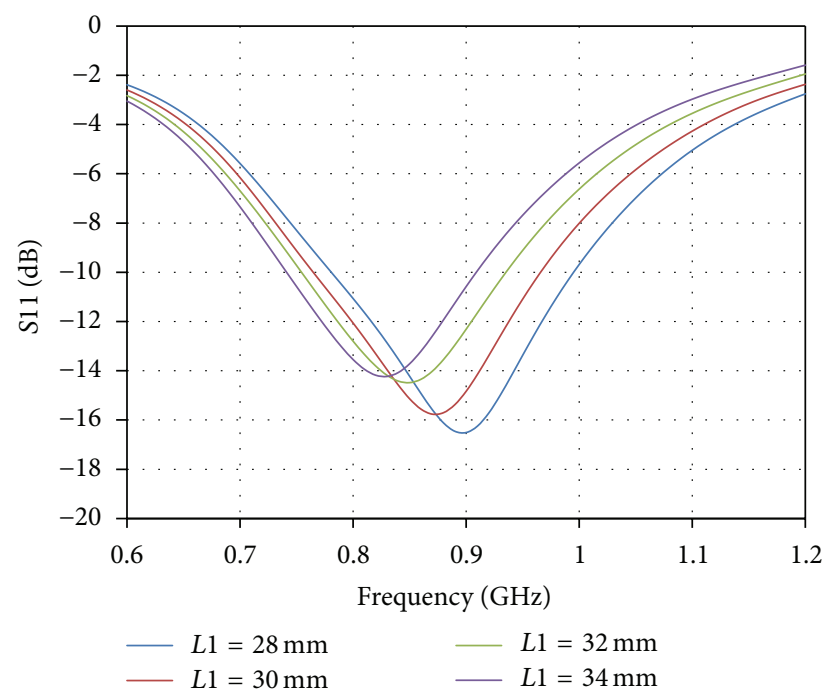

FIgURE 3: Parametric study on the influence of $L 1$.

The radiation patterns obtained when the 4 different radiating elements are independently fed are presented in Figure 10. For the sake of clarity, each radiation pattern is

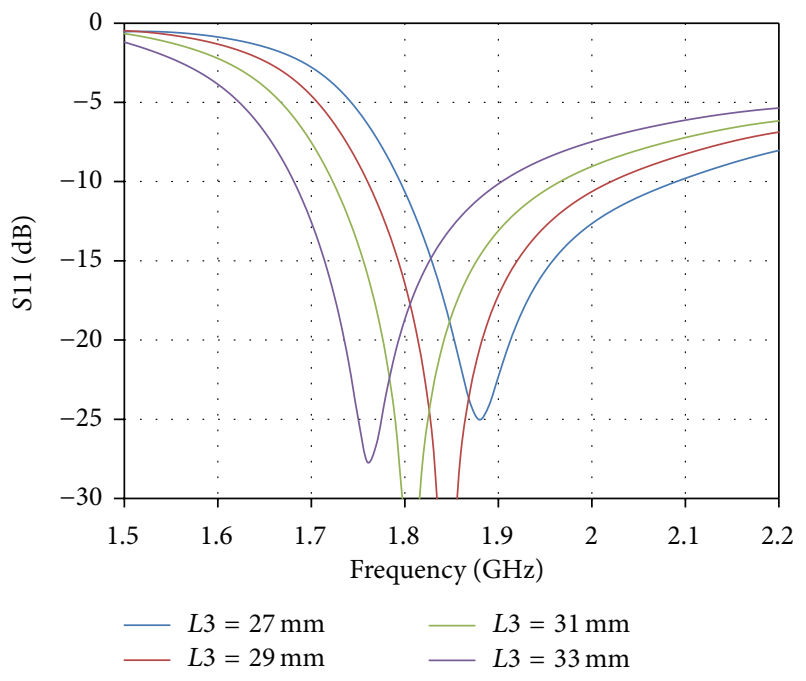

Figure 4: Parametric study on the influence of $L 3$.

placed on the corresponding excited antenna. It can be easily seen that the radiation pattern shapes are identical unless for a mirror symmetry around the chassis center. As widely 


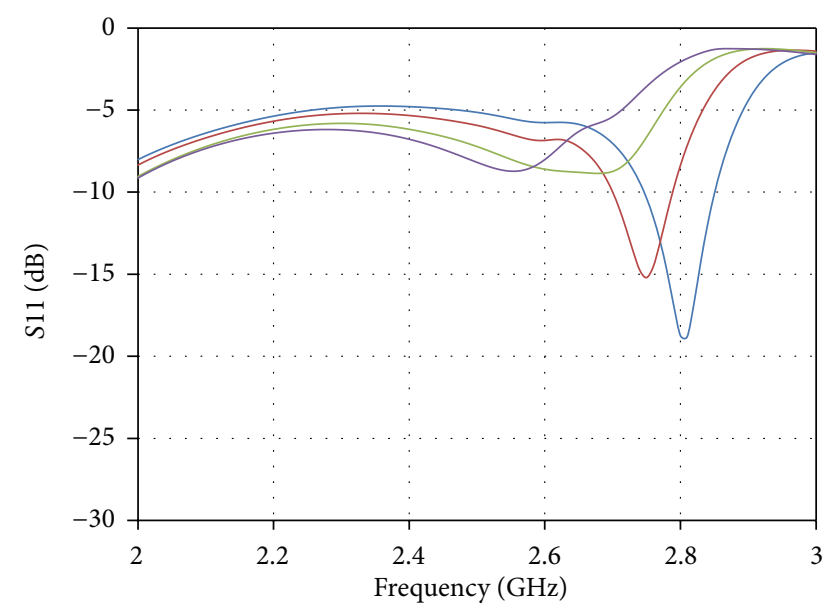

$\begin{array}{rlrl}H 3 & =5 \mathrm{~mm} \\ -H 3 & =6 \mathrm{~mm} & H 3 & =7 \mathrm{~mm} \\ -H 3 & =8 \mathrm{~mm}\end{array}$

FIgURE 5: Parametric study on the influence of $H 3$.

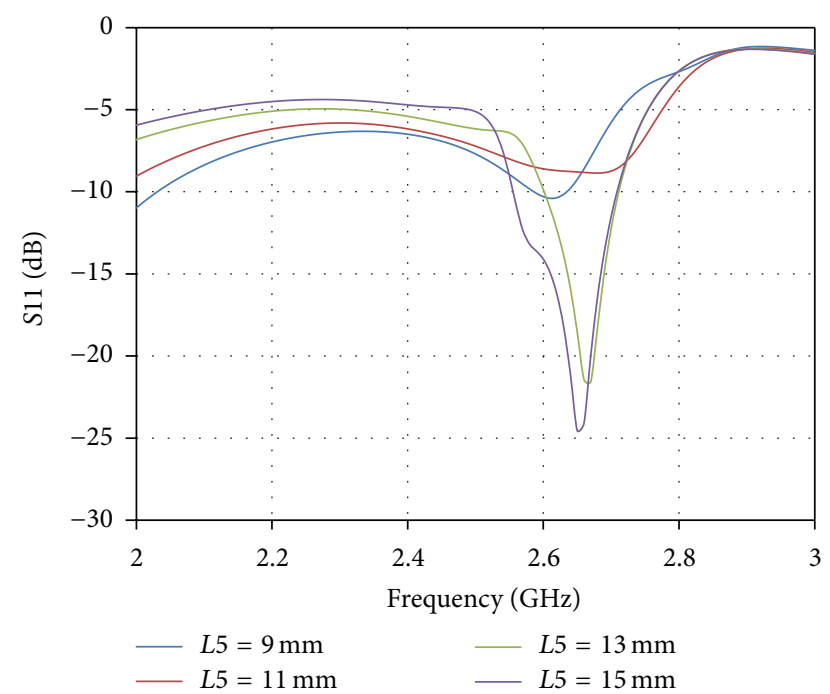

Figure 6: Parametric study on the influence of $L 5$.

known, two formulas can be used for the calculation of the ECC of MIMO antenna systems. The first is based on the $S$ parameters of the two antennas whereas the second (1) uses the 3D complex radiation patterns $\left[F_{1}(\theta, \phi)\right.$ and $\left.F_{2}(\theta, \phi)\right]$. Although much simpler to calculate, $S$-parameter equation is not suitable in this study since it may give misleading results since it does not take into account the efficiency losses of the antennas. For this reason, (1) (for an isotropic propagation environment) was used:

$$
\rho_{e}=\frac{\left|\iint_{4 \pi} F_{1}(\theta, \phi)^{*} \cdot F_{2}(\theta, \phi) d \Omega\right|^{2}}{\left(\iint_{4 \pi}\left|F_{1}(\theta, \phi)\right|^{2} d \Omega\right) \cdot\left(\iint_{4 \pi}\left|F_{2}(\theta, \phi)\right|^{2} d \Omega\right)} .
$$

The simulation of the ECC for the different antenna couples is presented in Table 2. The ECC values are always below 0.08 . Despite the smaller distance, it is worth noting that the antenna combination with the higher coupling (Antennas 1

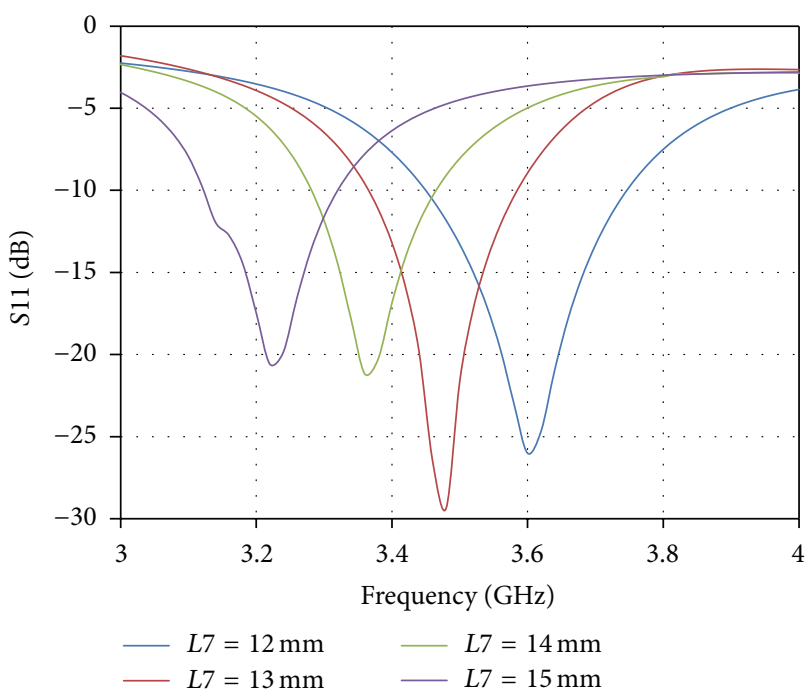

Figure 7: Parametric study on the influence of $L 7$.

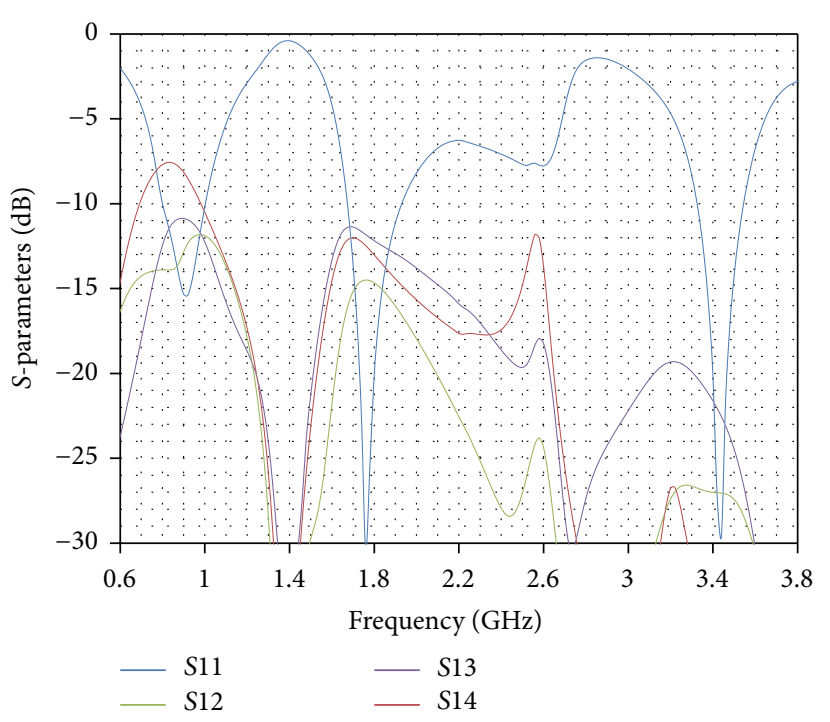

FIGURE 8: Simulated antenna system $S$-parameters.

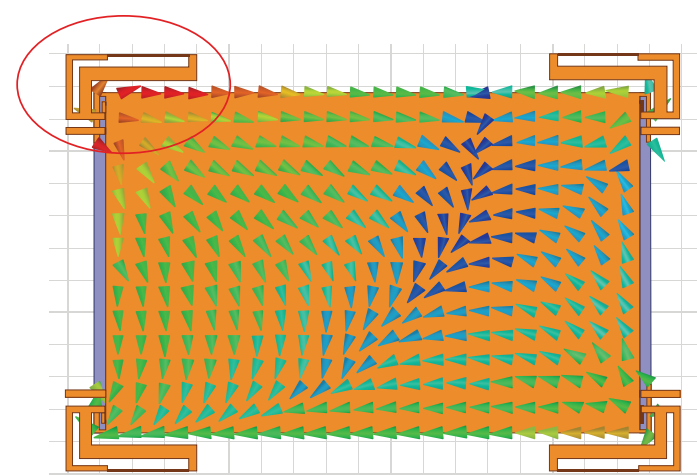

Figure 9: Current density on the chassis at $800 \mathrm{MHz}$ when Antenna 1 is fed. 
TABLE 2: Simulated ECC for the different antenna combinations.

\begin{tabular}{lcccc}
\hline ECC & $800 \mathrm{MHz}$ & $1.8 \mathrm{GHz}$ & $2.5 \mathrm{GHz}$ & $3.5 \mathrm{GHz}$ \\
\hline Antenna 1-Antenna 2 & 0.0058 & 0.0016 & 0.0026 & 0.00065 \\
Antenna 1-Antenna 3 & 0.0754 & 0.0094 & 0.0002 & 0.00035 \\
Antenna 1-Antenna 4 & 0.067 & 0.001 & 0.0087 & 0.0022 \\
\hline
\end{tabular}

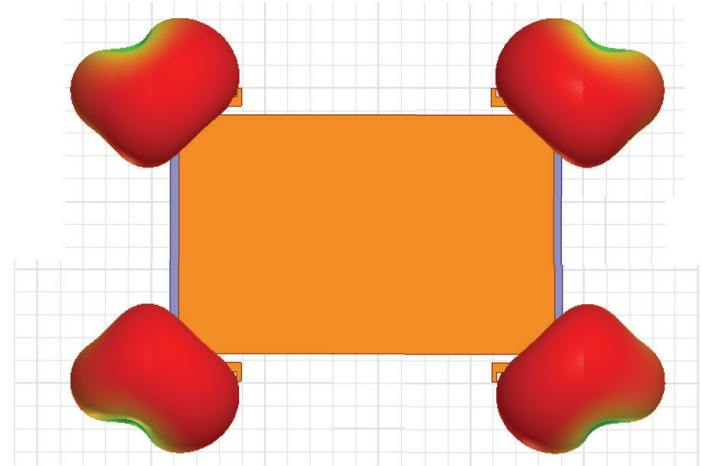

FIGURE 10: 3D radiation patterns at $800 \mathrm{MHz}$ when each antenna is independently fed.

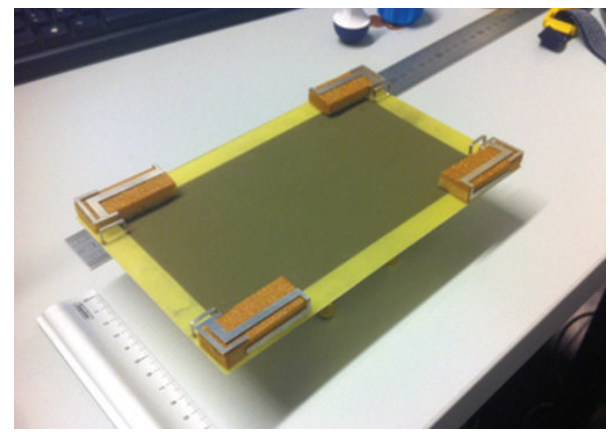

FIGURE 11: Antenna system prototype.

and 4) has a lower ECC than the antennas on the diagonal (Antennas 1 and 3). As a matter of fact, because of the symmetry, the radiation patterns of the antennas located on the same edge are less correlated than those of the antennas on the diagonal.

3.2. Prototype Measurements. According to the optimized parameters, a prototype of the antenna system was fabricated on a FR-4 Epoxy substrate of permittivity 4.4 and dimensions of $130 \times 190 \times 1.6 \mathrm{~mm}^{3}$. A picture of the prototype is shown in Figure 11.

From the measurements presented in Figure 12, it can be observed that the impedance bandwidth (defined for $S 11<-6 \mathrm{~dB}$ ) is $270 \mathrm{MHz}$ from $730 \mathrm{MHz}$ to $1000 \mathrm{MHz}$, corresponding to about $31 \%$ fractional bandwidth (FBW). In the middle band, the frequency bandwidth is $990 \mathrm{MHz}$, from $1710 \mathrm{MHz}$ to $2700 \mathrm{MHz}$ (45\% FBW). In the higher band, the antenna is matched with the same criteria from 3.3 to $3.65 \mathrm{GHz}(10 \% \mathrm{FBW})$.

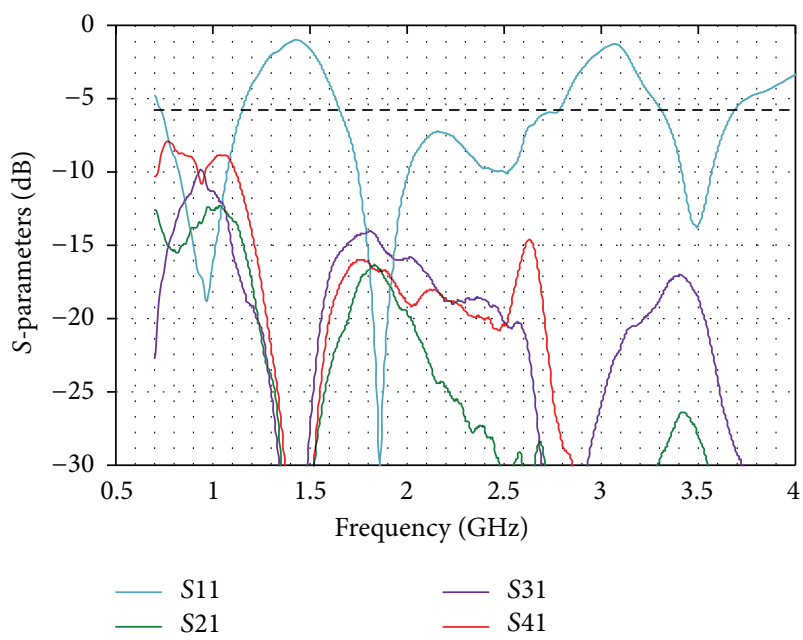

FIGURE 12: Measured S-parameters of the realized prototype.

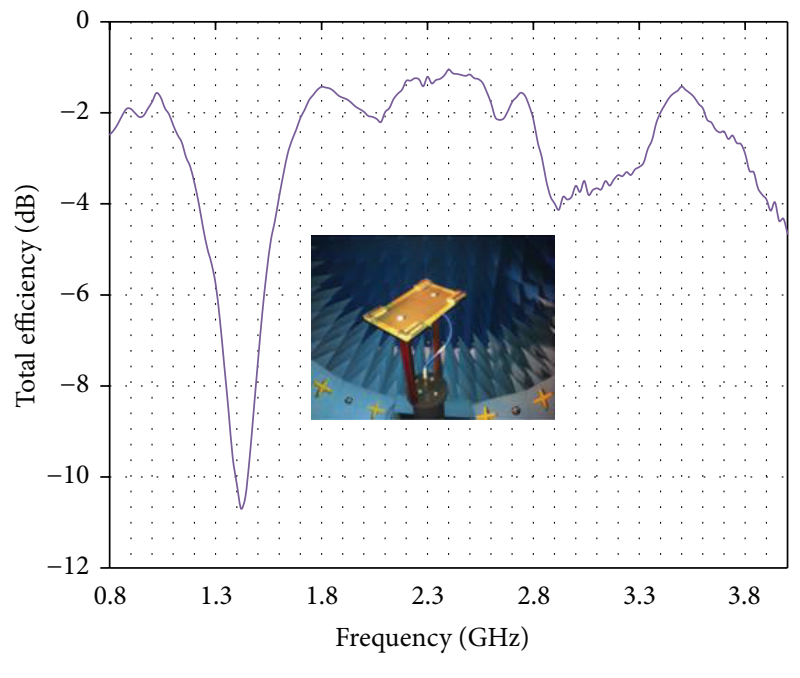

- Ant. 1

FIGURE 13: Measured total efficiency of the realized prototype.

The total efficiency of the prototype was also measured using a StarLab-Satimo station (Figure 13). For radiation measurement, the unused antenna ports were matched with a 50-ohm load. The measured antenna efficiency ranges from -2.7 to $-2 \mathrm{~dB}$ in the lower band, from -2.3 to $-1.8 \mathrm{~dB}$ in the middle band, and from -4 to $-2 \mathrm{~dB}$ in the higher band.

3.3. Influence of a Real PCB and Casing. In order to further validate the proposed solution with a realistic test bed, a commercial 7-inch tablet has been mechanically modified to integrate the optimized antenna elements. The measured Sparameters of the new antenna system prototype including the real PCB and the casing are presented in Figure 14. Some slight differences with respect to Figure 11 due to the coupling of the antennas with the electronic components on the PCB are observed. The measured prototype total efficiency (Figure 15) is acceptable and similar to what was 


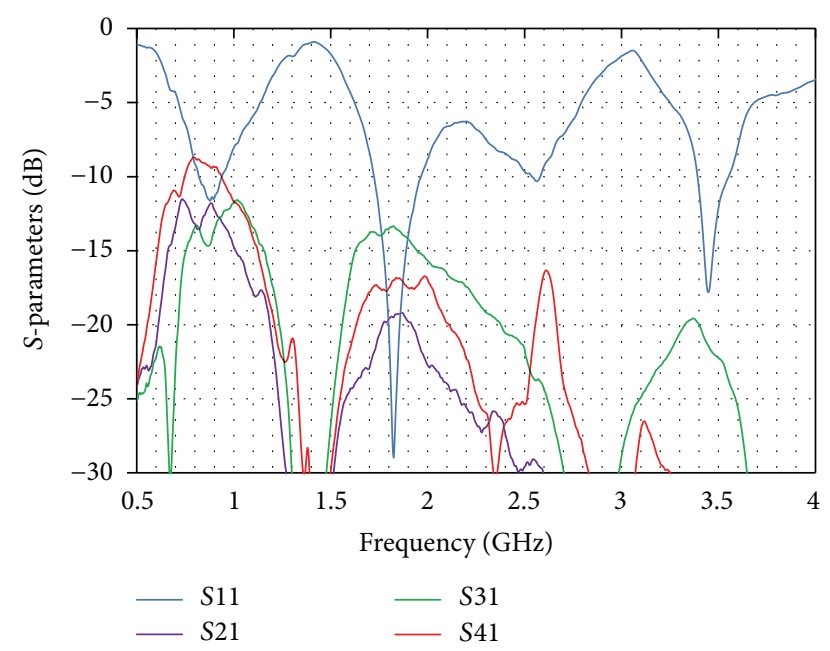

Figure 14: Measured S-parameters of the prototype in the realistic test bed.

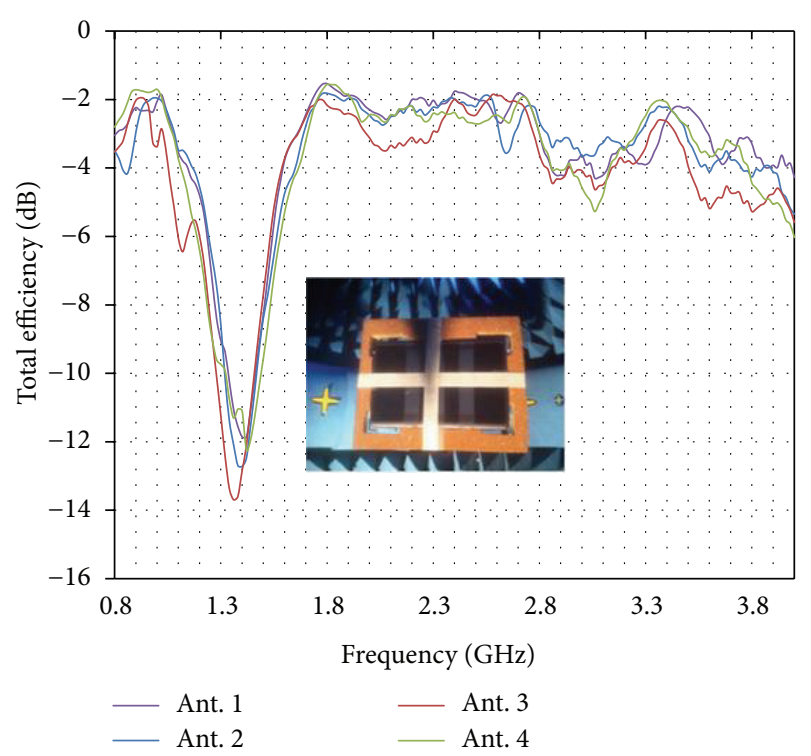

FIgURE 15: Measured total efficiency of the prototype in the realistic test bed.

shown in Figure 12. The nonsymmetrical placement of the electronic components causes small differences among the four antenna behaviors.

\section{Conclusion}

In conclusion, a multiband $4 \times 4 \mathrm{MIMO}$ antenna systems has been proposed. It can operate in the frequency bands usually required for compact mobile terminals including the low frequencies in the LTE standard (740-3650 MHz). The placement as well as the structure of the 4 antenna elements has been optimized in order to reduce the ECC. The proposed solution has been integrated into a commercial 7-inch tablet. The measured performance ensures that the antenna can operate with good performance over the required bands.

\section{Conflict of Interests}

The authors declare that there is no conflict of interests regarding the publication of this paper.

\section{Acknowledgments}

The authors would like to thank the CREMANT for its support. This work was realized in the framework of SPECTRA Celtic project.

\section{References}

[1] B. Mun, C. Jung, M.-J. Park, and B. Lee, "A compact frequencyreconfigurable multiband LTE MIMO antenna for laptop applications," IEEE Antennas and Wireless Propagation Letters, vol. 13, pp. 1389-1392, 2014.

[2] J. Ilvonen, R. Valkonen, J. Holopainen, and V. Viikari, “Design strategy for $4 \mathrm{G}$ handset antennas and a multiband hybrid antenna," IEEE Transactions on Antennas and Propagation, vol. 62, no. 4, pp. 1918-1927, 2014.

[3] A. Diallo, C. Luxey, P. Le Thuc, R. Staraj, and G. Kossiavas, "Enhanced diversity antennas for UMTS handsets," in Proceedings of the 1st European Conference on Antennas and Propagation (EuCAP '06), pp. 1-5, Nice, France, November 2006.

[4] W.-H. Hsu, C.-H. Wen, S.-C. Pan, and H.-Y. Jheng, "A tablet MIMO antenna with a wave-trap slot for LTE/WiMAX applications," in Proceedings of the International Symposium on Antennas and Propagation (ISAP '13), pp. 1307-1310, Nanjing, China, October 2013.

[5] S. Shoaib, I. Shoaib, N. Shoaib, C. Xiaodong, and C. G. Parini, "A $4 \times 4$ MIMO antenna system for mobile tablets," in Proceedings of the 8th European Conference on Antennas and Propagation (EuCAP '14), pp. 2813-2816, The Hague, The Netherlands, April 2014.

[6] F. Ferrero, A. Diallo, C. Luxey et al., "Two-element PIFA array structure for polarization diversity in UMTS mobile phones," Radioengineering, vol. 18, no. 4, pp. 407-412, 2009.

[7] R. Martens, E. Safin, and D. Manteuffel, "Selective excitation of characteristic modes on small terminals," in Proceedings of the 5th European Conference on Antennas and Propagation (EUCAP '11), pp. 2492-2496, IEEE, Rome, Italy, April 2011.

[8] P. Vainikainen, J. Ollikainen, O. Kivekäs, and I. Kelander, "Resonator-based analysis of the combination of mobile handset antenna and chassis," IEEE Transactions on Antennas and Propagation, vol. 50, no. 10, pp. 1433-1444, 2002.

[9] Y. Chen and C.-F. Wang, Characteristic Modes: Theory and Applications in Antenna Engineering, Wiley, New York, NY, USA, 2015. 

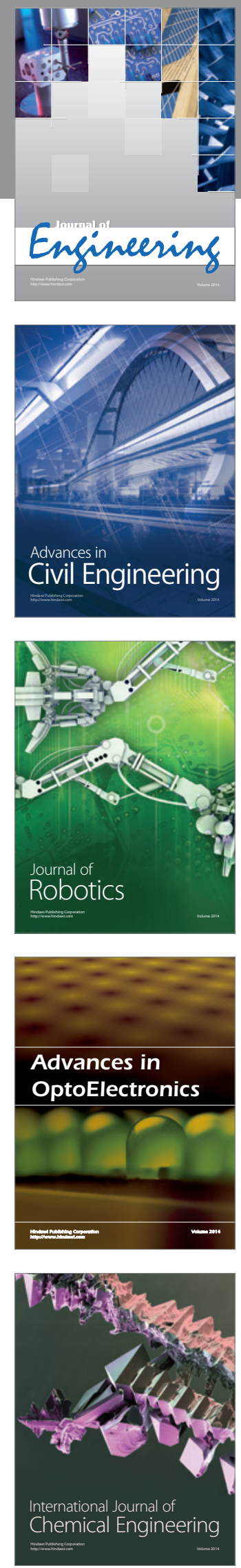

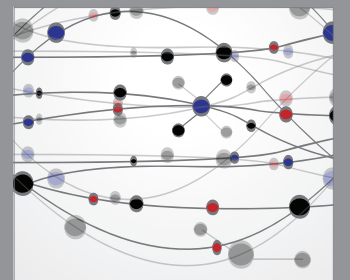

The Scientific World Journal
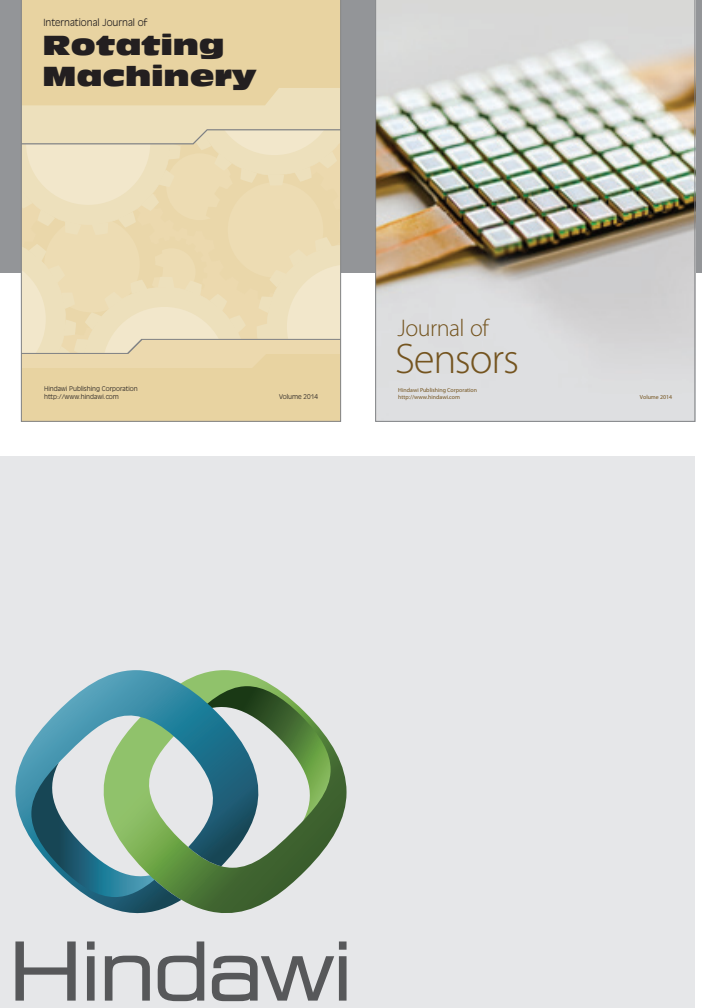

Submit your manuscripts at http://www.hindawi.com
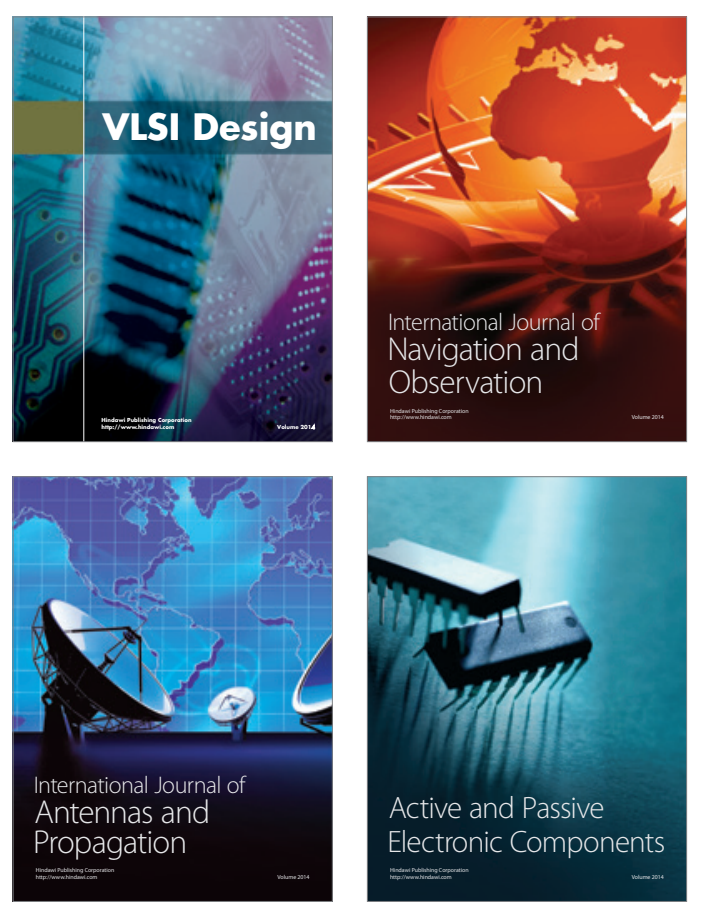
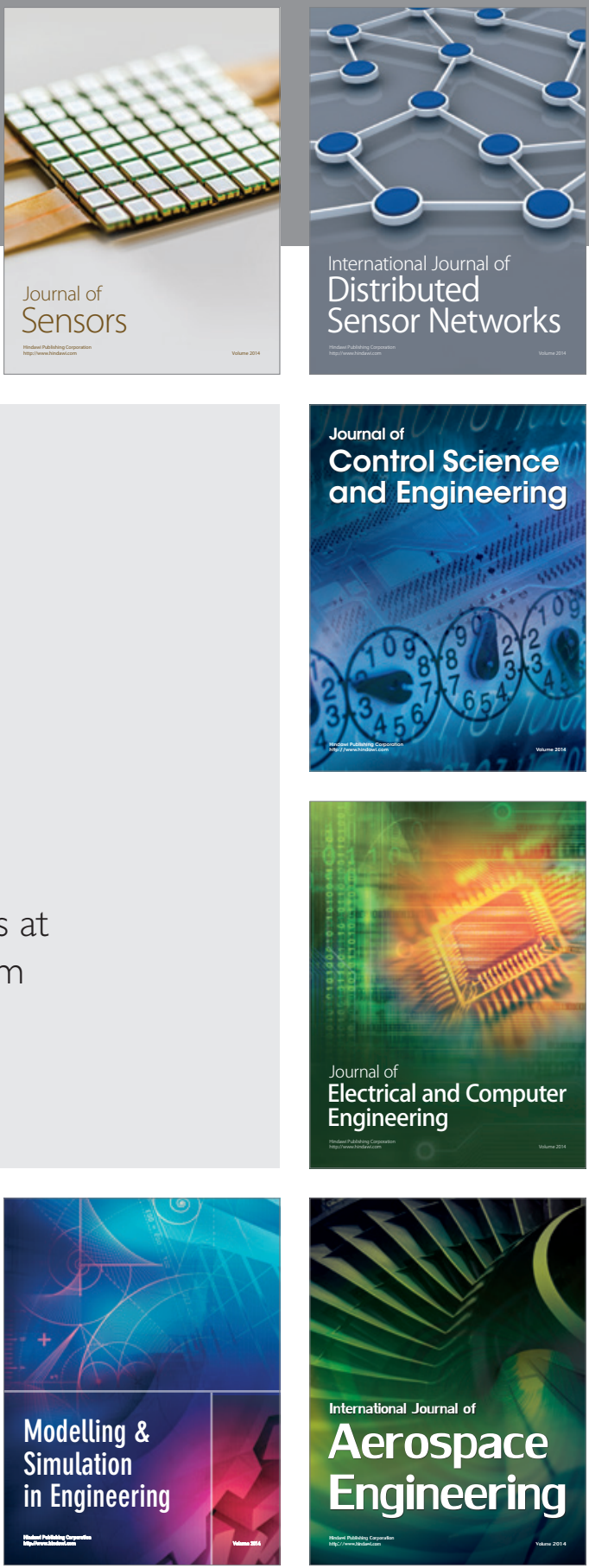

Journal of

Control Science

and Engineering
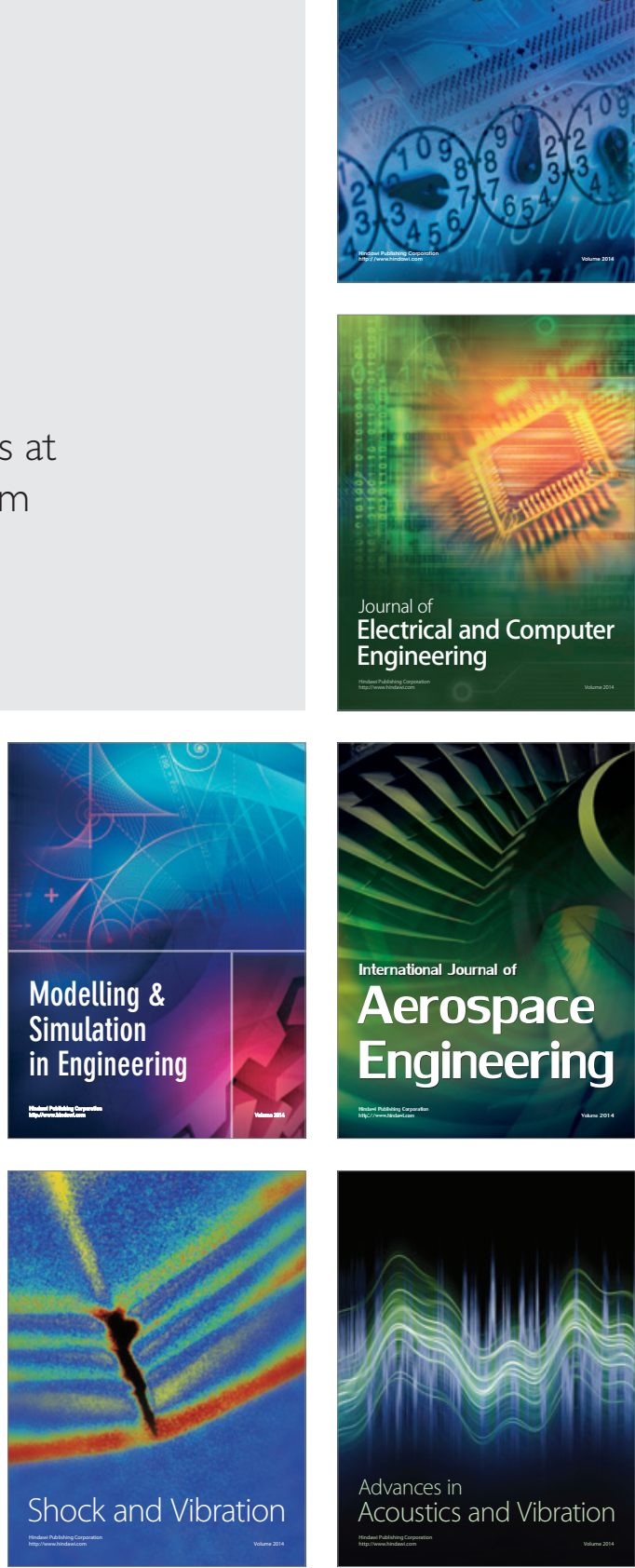